\title{
BMJ Open Characterising the long-term clinical outcomes of 1190 hospitalised patients with COVID-19 in New York City: a retrospective case series
}

\author{
Sherif M Shoucri (D) , ${ }^{1}$ Lawrence Purpura, ${ }^{1}$ Clare DeLaurentis, ${ }^{2}$ \\ Matthew A Adan (D) , ${ }^{3}$ Deborah A Theodore, ${ }^{1}$ Alexandria Lauren Irace (D) , ${ }^{3}$ \\ Shelief Y Robbins-Juarez, ${ }^{3}$ Apurva M Khedagi, ${ }^{3}$ Daniel Letchford, ${ }^{3}$ Amro A Harb, ${ }^{3}$ \\ Lillian M Zerihun, ${ }^{3}$ Kate E Lee (1) , ${ }^{3}$ Karen Gambina, ${ }^{3}$ Max C Lauring, ${ }^{3}$ Noah Chen, ${ }^{3}$ \\ Colin P Sperring, ${ }^{3}$ Sanket S Mehta, ${ }^{3}$ Ellen L Myers, ${ }^{3}$ Hueyjong Shih, ${ }^{3}$ \\ Michael G Argenziano (10, , ${ }^{3}$ Samuel L Bruce, ${ }^{3}$ Cody L Slater, ${ }^{3}$ Jonathan R Tiao, ${ }^{3}$ \\ Karthik Natarajan, ${ }^{4}$ George Hripcsak, ${ }^{4}$ Ruijun Chen, ${ }^{4,5}$ Michael T Yin, ${ }^{1}$ \\ Magdalena E Sobieszczyk, ${ }^{1}$ Delivette Castor, ${ }^{1}$ Jason E Zucker ${ }^{1}$
}

To cite: Shoucri SM, Purpura L, DeLaurentis C, et al. Characterising the long-term clinical outcomes of 1190 hospitalised patients with COVID-19 in New York City: a retrospective case series. BMJ Open 2021;11:e049488. doi:10.1136/ bmjopen-2021-049488

- Prepublication history and supplemental material for this paper is available online. To view these files, please visit the journal online (http://dx.doi org/10.1136/bmjopen-2021 049488).

Received 25 January 2021 Accepted 13 May 2021

Check for updates

(C) Author(s) (or their employer(s)) 2021. Re-use permitted under CC BY-NC. No commercial re-use. See rights and permissions. Published by BMJ.

For numbered affiliations see end of article.

Correspondence to Dr Sherif M Shoucri; sms2319@cumc.columbia.edu

\section{ABSTRACT}

Objective To characterise the long-term outcomes of patients with COVID-19 admitted to a large New York City medical centre at 3 and 6 months after hospitalisation and describe their healthcare usage, symptoms, morbidity and mortality.

Design Retrospective cohort through manual chart review of the electronic medical record.

Setting NewYork-Presbyterian/Columbia University Irving Medical Center, a quaternary care academic medical centre in New York City.

Participants The first 1190 consecutive patients with symptoms of COVID-19 who presented to the hospital for care between 1 March and 8 April 2020 and tested positive for SARS-CoV-2 on reverse transcriptase PCR assay.

Main outcome measures Type and frequency of follow-up encounters, self-reported symptoms, morbidity and mortality at 3 and 6 months after presentation, respectively; patient disposition information prior to admission, at discharge, and at 3 and 6 months after hospital presentation.

Results Of the 1190 reviewed patients, 929 survived their initial hospitalisation and 261 died. Among survivors, 570 had follow-up encounters (488 at 3 months and 364 at 6 months). An additional 33 patients died in the followup period. In the first 3 months after admission, most encounters were telehealth visits (59\%). Cardiopulmonary symptoms ( $35.7 \%$ and $28 \%$ ), especially dyspnoea $(22.1 \%$ and $15.9 \%)$, were the most common reported symptoms at 3-month and 6-month encounters, respectively. Additionally, a large number of patients reported generalised (26.4\%) or neuropsychiatric (24.2\%) symptoms 6 months after hospitalisation. Patients with severe COVID-19 were more likely to have reduced mobility, reduced independence or a new dialysis requirement in the 6 months after hospitalisation. Conclusions Patients hospitalised with SARS-CoV-2 infection reported persistent symptoms up to 6 months

\section{Strengths and limitations of this study}

This study reviews the 6-month outcomes of patients hospitalised with COVID-19 in the USA.

- The clinical characteristics and in-hospital outcomes of a subset of this cohort were previously reported early in the COVID-19 epidemic in the USA.

- Electronic health record data were abstracted for all available patients and were evaluated for persistent symptoms, morbidities and disposition during the follow-up period of interest.

- There was no active follow-up of patients, and as a result only 570 of the 929 survivors $(61 \%)$ during the study time had follow-up encounters at our institution, a limitation of these data.

- Lower follow-up rates could have resulted in selection and information bias if patients who were followed up were differential in key variables, resulting in either underestimation or overestimation of the prevalence of sequelae in this sample.

after diagnosis. These results highlight the long-term morbidity of COVID-19 and its burden on patients and healthcare resources.

\section{INTRODUCTION}

Since the first reports of a novel coronavirus identified in China in December 2019, SARS-CoV-2 has resulted in a global pandemic, with over 150 million cases and 3 million deaths globally as of May 2021. ${ }^{12}$ The disproportionate burden of infections has been in the USA. In May 2020, we reviewed the clinical characteristics and early outcomes of patients with a positive reverse transcriptase PCR (RT-PCR) assay result for SARS-CoV-2 
and described the in-hospital morbidity and mortality of patients presenting to NewYork-Presbyterian/Columbia University Irving Medical Center (NYP/CUIMC). ${ }^{3}$ However, much remains to be learnt about the long-term outcomes of this disease and the toll it takes on patients, their communities and the healthcare system in the weeks and months after diagnosis.

The long-term sequelae of COVID-19, now referred to as postacute sequelae of SARS-CoV-2 (PASC), have been increasingly recognised. ${ }^{4-11}$ Pulmonary and cardiovascular diseases are frequently reported long-term consequences of COVID-19. ${ }^{12-19}$ Neurological and neuropsychiatric sequelae, such as chronic pain, cognitive impairment, depression and anxiety, are also of concern and an active area of ongoing research. ${ }^{20-24}$ Studies of SARS-CoV-2 survivors done 30 days after symptom onset have shown persistent pulmonary abnormalities on CT of the chest. ${ }^{25}$ A case series of 143 patients in Rome, Italy found that 60 days after symptom onset, $87 \%$ of patients experienced at least one symptom; their prevalence in descending order were fatigue $(53.1 \%)$, dyspnoea $(43.4 \%)$, joint pain $(27.3 \%)$ and chest pain $(21.7 \%) .{ }^{26}$ In an observational cohort of hospitalised patients with SARS-CoV-2 infection in Michigan, USA, between March and July 2020, Chopra et $a l^{27}$ found that $33 \%$ of patients contacted 60 days after discharge $(\mathrm{n}=488)$ reported cardiopulmonary symptoms such as cough and dyspnoea. Furthermore, almost 50\% of patients felt emotionally affected by their health and $12 \%$ experienced new or worsening difficulty completing activities of daily living. Most recently, 6 months after acute infection, COVID-19 survivors in China ( $\mathrm{n}=1733)$ were found to have ongoing fatigue or muscle weakness $(63 \%)$, sleep difficulties $(26 \%)$, and anxiety or depression $(23 \%) .{ }^{11}$ The field has not yet characterised longerterm outcomes well.

We conducted a retrospective cohort study of previously hospitalised patients with COVID-19 to describe the occurrence and persistence of long-term sequelae of SARS-CoV-2 infection and the burden on the healthcare system. We aimed to characterise the frequency of ongoing symptoms and morbidity up to 6 months after presentation among surviving patients with follow-up encounters at our institution and describe potential correlates of PASC.

\section{METHODS}

\section{Data source and study sample}

The electronic medical records (EMR) of the first 1190 consecutive patients with symptoms of COVID-19 and subsequently diagnosed with SARS-CoV-2 infection by RT-PCR were examined. All patients were greater than 18 years old and hospitalised at NYP/CUIMC, a quaternary care medical centre in Northern Manhattan, New York City. Details on the early outcomes of this cohort were previously described. ${ }^{3}$ The baseline visit, defined as the visit when the patient first presented to the hospital, occurred between 1 March and 8 April 2020. Patients were identified in the institution's EMR, and their clinical data and laboratory results were abstracted into a REDCap database (Research Electronic Data Capture) instrument. ${ }^{28}{ }^{29}$ For patients with multiple hospital encounters for COVID-19 between 1 March and 30 April 2020, the visit representing their highest level of care (ie, emergency department (ED), hospital or intensive care unit (ICU) admission) or the last visit if the same level of care was delivered was selected for review. We elected to review all available unique records for this period rather than the initial cohort that comprised the first 1000 patients in the previous report. ${ }^{3}$ Beginning 26 March 2020, NYP/ CUIMC initiated a policy to provide all adult patients with COVID-19 who were able to be reached by telephone with a follow-up telehealth appointment on discharge. ${ }^{30}$ Patient consent was not applicable to this study as per CUIMC Institutional Review Board protocol.

\section{Manual chart review}

The REDCap instrument used for this study was linked to the survey from the prior study and included all baseline demographics, comorbidity information and oxygen requirements for patients at their initial COVID-19 hospital encounter. We manually abstracted data from the EMR for two follow-up time points representing 3 months and 6 months and baseline data for the additional patients identified in the study period. Three-month and six-month data were abstracted from every encounter occurring 1-92 days and 93-183 days after hospital presentation, respectively. We abstracted information on the following: vital statistics (ie, dead or alive), types of encounters, dates of encounters, setting (telehealth vs inperson), presence of symptoms, morbidity outcomes and disposition at follow-up. For patients with multiple encounters in each time point, presence of symptoms and morbidity outcomes were assessed for persistence. Persistence was defined as the presence of symptoms or morbidity outcomes without reported resolution throughout each 3-month observation period. Symptoms or morbidity outcomes that resolved were defined as nonpersistent. For patients without documented encounters, the follow-up period was defined as missing. If a patient died during the baseline hospital visit or by 3 months, their data were censored at the visit representing their death. For patients who remained hospitalised for more than 92 days from their initial COVID-19 presentation, data were only abstracted at the 6-month time point. Only follow-up encounters documented in the NYP/CUIMC EMR were included in the review.

An abstraction team of 19 medical students from the Columbia University Vagelos College of Physicians and Surgeons, supervised by research clinicians, manually abstracted data from the EMR. Abstractors were trained on data entry as the first step in data quality assurance (DQA). The comprehensive DQA included data verification through a random sampling of records by the lead clinician in addition to regular team meetings. Additionally, abstractors flagged all records with inconsistencies for 
a secondary review by the lead clinician, who reconciled any discrepancies. As a final step in DQA, an abstractor dedicated to quality control reviewed charts identified by the lead clinician to resolve any remaining issues.

\section{Variable definitions}

An encounter was defined as any ED visit, inpatient hospitalisation or scheduled outpatient encounter. Outpatient encounter types were categorised as outpatient primary care, rehabilitation, specialty or mental health. Encounters were labelled as telehealth visits if they were documented as a telemedicine, video or telephone visit. Telephone contact notes documented in the EMR were not treated as encounters if the patient did not participate, although this information was used to ascertain patient disposition information and morbidity outcomes.

Symptoms not explicitly reported or documented were assumed not present. Certain symptoms were classified into symptom group clusters, including fevers (fever, chills, night sweats); myalgias or arthralgias (myalgias, arthralgias, non-specific body aches, leg pain or cramps); altered mentation (confusion, loss of memory, altered mental status); and urinary symptoms (incontinence, frequency, urgency, dysuria). Four larger symptom groups were evaluated: cardiopulmonary (dyspnoea, cough, chest pain, lower extremity oedema); generalised (fevers, myalgias or arthralgias, fatigue, anosmia or ageusia); neuropsychiatric (weakness, altered mentation, gait imbalance, depression or anxiety, headache); and gastrointestinal or urinary (abdominal pain, nausea or vomiting, diarrhoea, constipation, urinary symptoms).

Five morbidity outcomes were evaluated: new oxygen requirement (or an increase from baseline if previously on oxygen), reduced mobility, reduced independence, new dialysis dependence or no morbidity. Reduced mobility was based on the need for an ambulatory assist device, ongoing physical therapy or reported difficulty with ambulation. Reduced independence was defined as the inability to live alone without support from a home health aide, nurse or family member. COVID-19 severity was categorised by the maximal amount of oxygen supplementation required during the initial hospital encounter. Mild disease was defined as patients who did not need supplemental oxygen or required oxygen via nasal cannula or venturi mask. Moderate disease included patients who needed oxygen via a non-rebreather mask, high-flow nasal cannula or non-invasive ventilation. Patients who required intubation were defined as having severe disease. Morbidity outcomes were assessed any time in the 6 months (183 days) after hospital admission.

Disposition was defined as patients' living location and status in the community, and was evaluated on admission, discharge and at 3 and 6 months. Five dispositions were reported: living at home, living in a nursing or rehabilitation facility, hospitalised, undomiciled or residing in a homeless shelter, and dead or receiving hospice care. For patients with multiple encounters, the last known disposition was reported.

\section{Data analysis}

Descriptive analyses were conducted for baseline (presenting) and each follow-up visit. For categorical variables, frequency counts and proportions were calculated at baseline and each follow-up period. For continuous variables, median and IQR were calculated. Selection bias was also ascertained through descriptive analyses by follow-up status (only baseline vs any follow-up visit), comorbidity status (any and type of comorbidity at baseline vs none) and hospital surveillance approach period (before and after 26 March 2020, representing more active monitoring of patient outcomes). All analyses were performed using R V.4.0.3. ${ }^{31}$

\section{Patient and public involvement statement}

Patients and the public were not directly involved in the design of this study. The retrospective nature of the study relied on chart reviews, thus limiting participation to hospital personnel. With the second wave of SARS-CoV-2 infections on us, the timely characterisation of outcome data further limited patient and public involvement.

\section{RESULTS}

The first 1190 patients who tested positive for SARS-CoV-2 infection at NYP/CUIMC presented between 1 March and 8 April 2020. During the initial presentation, 261 patients died, leaving 929 to be subsequently followed and included in this analysis. Of the survivors, 570 patients had at least one follow-up encounter at our institution, while 359 had no encounter (figure 1). Among patients with encounters, 282 patients had encounters at both 3 months and 6 months, while 206 and 82 had encounters at 3 months and 6 months only, respectively. The median number of days from hospital presentation to follow-up visit was 67 days (IQR 36-82) in the first 3 months and 158 days (IQR 133-174) in the next 3 months.

Baseline and longitudinal characteristics of this cohort are shown in table 1. Among patients with encounters in each 3-month observation period, age, gender, race and body mass index were comparable. The most frequently reported comorbidities are presented in table 1. A full listing of all patient comorbidities is available in online supplemental table 1 . The proportion of major medical comorbidities at baseline and at each 3-month follow-up also remained overall stable. Notable exceptions were dementia, cerebrovascular accident (CVA), malignancy and pulmonary disease. Patients with dementia and CVA had fewer follow-up encounters, while patients with malignancy and baseline pulmonary disease had more clinical encounters at the 6-month follow-up. To examine possible selection bias and its effect on observed outcomes, the baseline characteristics of patients with and without follow-up encounters were compared (online supplemental table 2). Notably, patients with follow-up encounters had a higher frequency of medical comorbidities, ICU admissions and intubations. 


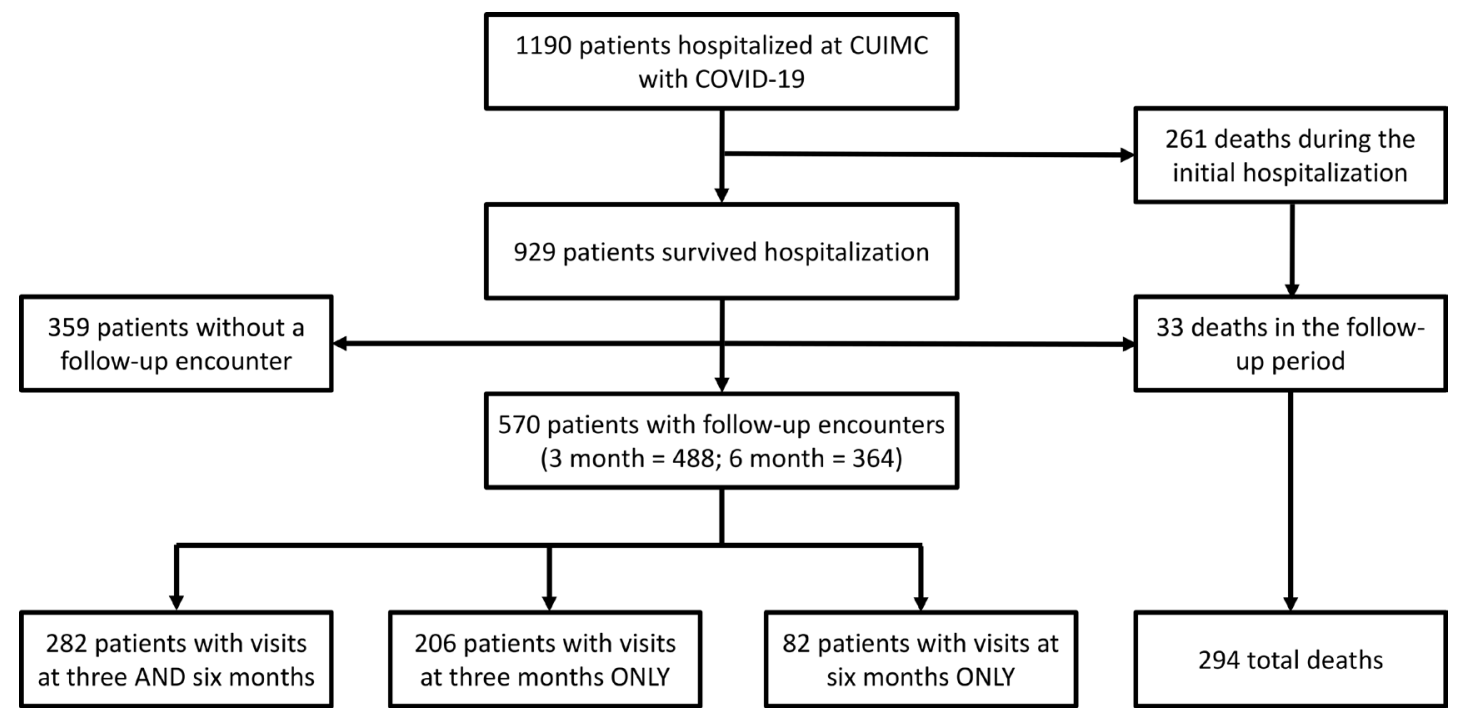

Figure 1 Study flow diagram. CUIMC, Columbia University Irving Medical Center.

The majority of follow-up visits in the first 3 months after COVID-19 hospitalisation were performed remotely (59\%), and this proportion declined to $28.2 \%$ by 6 months (table 2). Visits to specialists were the most common encounter during each follow-up period in both groups. Approximately one-third of visits in the first 3 months were to an outpatient primary care provider (PCP); at 6 months, the percentage of PCP visits dropped to $18.7 \%$. The frequency of inpatient admissions $(7.1 \%$ vs $6.3 \%)$ and $\mathrm{ED}$ visits $(9.3 \%$ vs $10.1 \%)$ was similar at both times. Overall, there were very few mental health visits after COVID-19 hospitalisation at both $3(1.6 \%)$ and $6(1.8 \%)$ months. Follow-up rates for patients discharged before and after the 26 March 2020 initiation of a hospital-wide process providing discharge appointments are presented in online supplemental table 3. Both rates of follow-up (57\% vs 62\%) and number of follow-up visits (1.0 (0.4) vs $1.0(0.4))$ were equivalent for patients discharged before or after this date.

Among the 570 patients with follow-up encounters, numerous symptoms persisted long after the initial hospital presentation for COVID-19 (table 3). The most common symptoms on admission (fever, cough and dyspnoea) all significantly decreased in the months after diagnosis; however, $28 \%$ of patients experienced cardiopulmonary symptoms 6 months after their diagnosis. Dyspnoea, the most common symptom in the follow-up period, was present in $22.1 \%$ of patients at 3 months and remained in $15.9 \%$ of patients at 6 months. The percentage of patients reporting cough (16\% and $10.2 \%)$, weakness $(8.4 \%$ and $9.3 \%)$ or fatigue $(9 \%$ and $10.4 \%)$ also remained elevated at 3 and 6 months, respectively. At 6 months, abdominal pain $(9.3 \%)$, gait instability $(4.9 \%)$, and myalgias or arthralgias $(17.6 \%)$ occurred at a higher frequency when compared with the first 3-month interval $(5.1 \%, 1.8 \%$ and $11.1 \%$, respectively). The frequency of most neurological and neuropsychiatric symptoms did not decrease with time, including headache, altered mentation, and depression or anxiety. Most symptoms reported at 3 months occurred at a higher frequency in patients with a history of any comorbidity than those without comorbidities (online supplemental table 4). At 6 months, the percentages of patients with cardiopulmonary (29\% vs $25 \%$ ), generalised ( $27 \%$ vs $25 \%$ ), and gastrointestinal or urinary (20\% vs $21 \%$ ) symptoms were similar between patients with or without comorbidities, respectively. Neuropsychiatric symptoms were more common in patients with comorbidities $(25 \%)$ than without $(18 \%)$.

Morbidity outcomes were reported on 600 of the 929 survivors. Patients with moderate and severe COVID-19 suffered from increased morbidity in the 6 months after their initial hospitalisation (table 4). One in five patients had reduced mobility or reduced independence 6 months later, and $60.2 \%$ of patients with severe COVID-19 had reduced mobility. New or increased oxygen requirements from baseline were noted in $8.3 \%$ of patients, most of whom either had moderate or severe disease. An additional $33(5.5 \%)$ died in the follow-up period, 29 at 3 months and 4 at 6 months.

Disposition information was available for all 1190 patients on hospital admission and discharge, 574 patients at 3 months and 445 patients at 6 months (table 5). Although only $7.2 \%$ of patients presented to the hospital from a nursing or rehabilitation facility, $13.2 \%$ were discharged to one. Among survivors, however, 17\% (157 of 929) were discharged to a nursing or rehabilitation facility. Further, $10.3 \%$ of patients remained in a nursing or rehabilitation facility 3 months after their COVID-19 hospitalisation. Patients were therefore more likely to be located at home at 6 months $(86.8 \%, 386$ of 445) compared with 3 months $(79.1 \%, 454$ of 574$)$.

\section{DISCUSSION}

In this retrospective study of the first 1190 consecutive hospitalised patients infected with SARS-CoV-2 at NYP/CUIMC, 
Table 1 Baseline characteristics of patients from their initial hospitalisation for COVID-19 and at 3-month and 6-month followup encounters

\begin{tabular}{|c|c|c|c|}
\hline Characteristics & $\begin{array}{l}\text { Initial hospitalisation } \\
\mathrm{N}=1190(\%)\end{array}$ & $\begin{array}{l}3 \text { months } \\
\mathrm{n}=488(\%)\end{array}$ & $\begin{array}{l}6 \text { months } \\
\mathrm{n}=364(\%)\end{array}$ \\
\hline \multicolumn{4}{|l|}{ Follow-up encounter information } \\
\hline Days to follow-up encounter* & $127(59-166)$ & $67(36-82)$ & $158(133-174)$ \\
\hline Number of follow-up encounters* & $0.0(0.0-3.0)$ & $2.0(1.0-4.0)$ & $2.0(1.0-5.0)$ \\
\hline \multicolumn{4}{|l|}{ Baseline demographics } \\
\hline $\mathrm{Age}^{*}$ & $63(51-75)$ & $60(47.8-71)$ & $61(50.0-71)$ \\
\hline $18-34$ & $97(8.2)$ & $54(11.1)$ & $30(8.2)$ \\
\hline $35-54$ & 277 (23.2) & $127(26.0)$ & $96(26.4)$ \\
\hline $55-64$ & $250(21.0)$ & $120(24.6)$ & $95(26.1)$ \\
\hline$>65$ & $566(47.6)$ & $187(38.3)$ & $143(39.3)$ \\
\hline $\mathrm{BMI}^{*}$ & $28(25-33)$ & $28(25-33)$ & $29(25-33)$ \\
\hline \multicolumn{4}{|l|}{ Sex at birth } \\
\hline Male & 705 (59.2) & $277(56.8)$ & $190(52.2)$ \\
\hline Female & $485(40.8)$ & $211(43.2)$ & $174(47.8)$ \\
\hline \multicolumn{4}{|l|}{ Race and ethnicity } \\
\hline Hispanic or Latinx & $582(48.9)$ & $233(47.7)$ & $189(51.9)$ \\
\hline Other & $430(36.1)$ & $162(33.2)$ & $127(34.9)$ \\
\hline Not specified & $311(26.1)$ & $132(27.0)$ & $81(22.3)$ \\
\hline Black & $220(18.5)$ & $102(20.9)$ & $76(20.9)$ \\
\hline White & $209(17.6)$ & $85(17.4)$ & $74(20.3)$ \\
\hline Asian & $20(1.7)$ & $7(1.4)$ & $6(1.6)$ \\
\hline \multicolumn{4}{|l|}{ Smoking status } \\
\hline Never smoker & $910(76.4)$ & $367(75.2)$ & $262(71.9)$ \\
\hline Former smoker & $222(18.7)$ & 96 (19.7) & 81 (22.3) \\
\hline Active smoker & $55(4.6)$ & $25(5.1)$ & $21(5.8)$ \\
\hline \multicolumn{4}{|l|}{ Medical comorbidities } \\
\hline Hypertension & $717(60.3)$ & $277(56.8)$ & $225(61.8)$ \\
\hline Diabetes mellitus & $451(37.9)$ & $171(35.0)$ & $134(36.8)$ \\
\hline Hyperlipidaemia & $393(33.0)$ & $158(32.4)$ & $125(34.3)$ \\
\hline COPD or asthma & $202(17.0)$ & $87(17.8)$ & $74(20.3)$ \\
\hline Coronary artery disease & $153(12.9)$ & $58(11.9)$ & $41(11.3)$ \\
\hline Dementia & $125(10.5)$ & $32(6.6)$ & $14(3.8)$ \\
\hline Congestive heart failure & $124(10.4)$ & $46(9.4)$ & $41(11.3)$ \\
\hline Chronic kidney disease & $106(8.9)$ & $41(8.4)$ & $29(8.0)$ \\
\hline Cerebrovascular accident & $100(8.4)$ & $32(6.6)$ & $21(5.8)$ \\
\hline Other pulmonary condition & $77(6.5)$ & $46(9.4)$ & $41(11.3)$ \\
\hline Malignancy & $73(6.1)$ & $35(7.2)$ & $32(8.8)$ \\
\hline \multicolumn{4}{|l|}{ Disposition at initial hospitalisation } \\
\hline Emergency department visit & $167(14.0)$ & $67(13.7)$ & 48 (13.2) \\
\hline Hospital admission & $735(61.8)$ & $330(67.6)$ & $239(65.7)$ \\
\hline ICU admission & $288(24.2)$ & $91(18.6)$ & $77(21.1)$ \\
\hline \multicolumn{4}{|l|}{ Maximum oxygen requirement } \\
\hline No supplemental oxygen & $314(26.4)$ & $173(35.5)$ & $118(32.4)$ \\
\hline Nasal cannula or venturi mask & $342(28.7)$ & $145(29.7)$ & $105(28.8)$ \\
\hline
\end{tabular}




\begin{tabular}{|c|c|c|c|}
\hline Characteristics & $\begin{array}{l}\text { Initial hospitalisation } \\
\mathrm{N}=1190(\%)\end{array}$ & $\begin{array}{l}3 \text { months } \\
\mathrm{n}=488(\%)\end{array}$ & $\begin{array}{l}6 \text { months } \\
\mathrm{n}=364(\%)\end{array}$ \\
\hline HFNC or non-rebreather mask & $235(19.7)$ & $84(17.2)$ & $64(17.6)$ \\
\hline Non-invasive ventilation & $12(1.0)$ & $3(0.6)$ & $2(0.5)$ \\
\hline Intubation & $287(24.1)$ & $83(17.0)$ & $75(20.6)$ \\
\hline Death & $261(21.9)$ & & \\
\hline
\end{tabular}

*Statistics presented: median (IQR).

BMI, body mass index; COPD, chronic obstructive pulmonary disease; HFNC, high-flow nasal cannula; ICU, intensive care unit.

we found that cardiopulmonary complaints were the most prevalent symptom reported in the 6 months after hospital presentation, although both generalised and neuropsychiatric symptoms also persisted over the follow-up period. Additionally, we found that morbidity was associated with severity of initial illness up to 6 months after hospital presentation. Reduced independence $(51.7 \%)$ or reduced mobility $(60.2 \%)$ was present in over half of all patients who required intubation. Furthermore, $13 \%$ of patients were discharged to a nursing or rehabilitation facility, while only $7 \%$ of patients were initially admitted from such a facility. Our study also revealed the modes and types of healthcare encounters that these survivors had during the 6 months after discharge. Follow-up within the first 3 months was mostly via telehealth encounters (59\%), while inperson encounters became more prevalent at 6 months $(71.8 \%)$.

This study evaluated patient outcomes up to 6months after their initial hospitalisation for COVID-19. Published reports to date have characterised symptoms in COVID-19 survivors in the first few months after illness, and like ours identified pulmonary manifestations such as dyspnoea and cough as dominant symptoms. Interestingly, in an Italian cohort of 143 patients, $43 \%$ reported dyspnoea around 60 days post discharge, while in 55 patients in China only $14 \%$ had exertional dyspnoea at 3 months. ${ }^{26}{ }^{32}$ Both these studies included individuals with less severe disease manifestations, with only $5 \%$ requiring intubation in the Italian study and

\begin{tabular}{lcc}
\hline $\begin{array}{l}\text { Table } 2 \\
\text { up encounters }\end{array}$ & $\begin{array}{l}\text { Encounter information among patients with follow- } \\
\mathbf{n = 1 3 4 5}(\%)\end{array}$ & $\begin{array}{l}\mathbf{6} \text { months } \\
\mathbf{n}=\mathbf{1 1 9 8}(\%)\end{array}$ \\
\hline Characteristics & & \\
\hline Encounter type & $125(9.3)$ & $121(10.1)$ \\
\hline Emergency department visit & $96(7.1)$ & $76(6.3)$ \\
\hline Inpatient admission & $472(35.1)$ & $224(18.7)$ \\
\hline Outpatient primary care visit & $587(43.6)$ & $681(56.8)$ \\
\hline Specialty visit & $43(3.2)$ & $74(6.2)$ \\
\hline Rehabilitation visit & $22(1.6)$ & $22(1.8)$ \\
\hline Mental health visit & & \\
\hline Encounter setting & $552(41.0)$ & $860(71.8)$ \\
\hline Inperson & $793(59.0)$ & $338(28.2)$ \\
\hline Telehealth &
\end{tabular}

less than $1 \%$ requiring supplemental oxygen in the Chinese cohort, suggesting that even patients with mild disease may suffer from persistent symptoms. Our finding of persistent neuropsychiatric symptoms 6 months after COVID-19 hospitalisation highlights the need for further investigation into this association. ${ }^{20} 21$ Since this was an observational study, we examined the role that confounding, selection and information bias might play in our observed findings and found that, although patients with comorbidities tended to have had more follow-up visits than those without, the prevalence of symptoms at 6 months did not differ by presence or absence of comorbidities at baseline. These findings support that these sequelae can occur in previously healthy patients at comparable levels and are not driven by pre-existing conditions.

We found that patients with severe COVID-19 were much more likely to report reduced mobility or independence in the 6 months after their hospitalisation. Along with decreased functional status, $8 \%$ of survivors required supplemental oxygen in the 6 months after presentation; this represented $15 \%$ in those with moderate or severe disease. These findings highlight the ongoing burden of this disease at the individual level; additionally, impact on the community level is illustrated by our finding that one in eight patients, and up to one in six survivors $(17 \%, 157$ of 929), were discharged to a nursing home or rehabilitation centre. Previous studies of survivors of acute respiratory distress syndrome (ARDS) have found persistent functional limitations 1 year after ICU discharge, suggesting that the morbidity associated with severe COVID-19 may continue even longer into the follow-up period. ${ }^{33}$ In contrast, in another study of patients with ARDS due to the 2009 influenza A (H1N1) virus pandemic, 1year after their ICU discharge survivors had only minor lung disabilities and most had returned to work. ${ }^{34}$ Nevertheless, our findings up to 6 months after presentation suggest that communities and healthcare systems should prepare for an increased number of patients who require nursing care and rehabilitation after hospitalisation with COVID-19. The creation of dedicated clinics for patients with PASC, staffed by clinicians with expertise in the long-term sequelae of the disease, would represent a sensible first step in addressing this need.

Telehealth visits accounted for $59 \%$ of health encounters in the first 3 months after hospital presentation. This aligned with a prior study of patients with COVID-19 in Michigan where $79 \%$ of follow-up within the first 60 days occurred via 
Table 3 Persistent symptoms* reported for patients with follow-up encounters

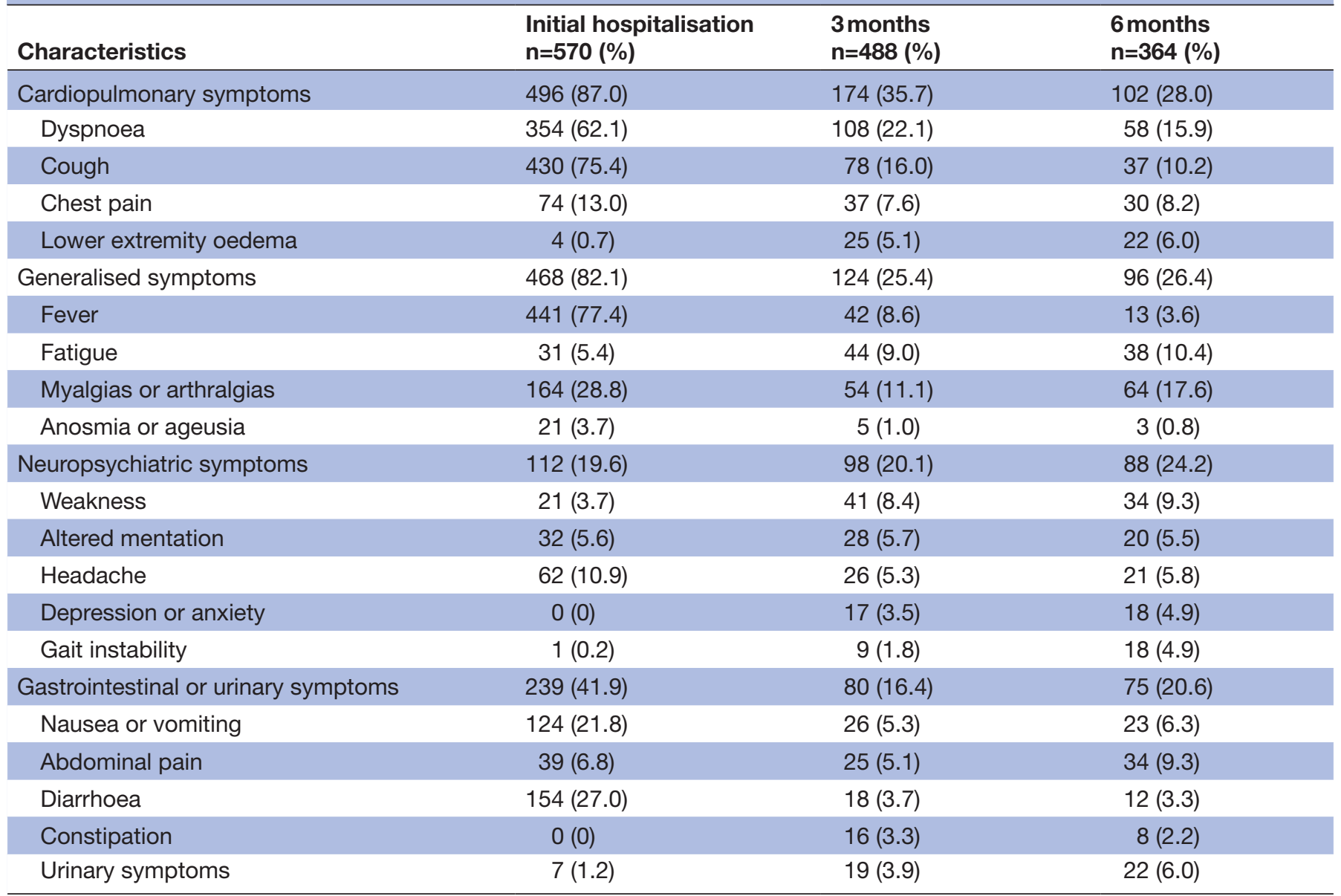

${ }^{*}$ Persistent symptoms are symptoms that did not resolve during each 3-month follow-up period.

telephone or video visit. ${ }^{27}$ Given that the follow-up period of this study ranged from March to October, early telehealth visits were likely influenced by the spring lockdown of New York City. Many clinics were closed and prioritised telehealth visits in order to minimise non-essential travel and inperson interactions. As the city reopened in the summer, so too did most clinics, and thus the majority of encounters were in person; however, over a quarter of visits continued to take place remotely. As SARS-CoV-2 infections surge across the globe, these findings validate the key role of telehealth visits during a pandemic and the need for healthcare organisations to prepare for widespread implementation of telemedicine capabilities. ${ }^{35}$ Further, the sustained application of telemedicine highlights the importance of continued research into

Table 4 Outcomes up to 6 months after hospitalisation among patients who survived, stratified by COVID-19 severity*

\begin{tabular}{|c|c|c|c|c|}
\hline & Total & Mild & Moderate & Severe \\
\hline Characteristics & $\mathrm{n}=600(\%)$ & $\mathrm{n}=379(\%)$ & $n=103(\%)$ & $n=118(\%)$ \\
\hline No morbidity & $389(64.8)$ & $299(78.9)$ & $63(61.2)$ & $27(22.9)$ \\
\hline Reduced mobility† & $124(20.7)$ & $34(9)$ & $19(18.4)$ & $71(60.2)$ \\
\hline Reduced independenceł & $116(19.3)$ & $35(9.2)$ & $20(19.4)$ & $61(51.7)$ \\
\hline Death & $33(5.5)$ & $23(6.1)$ & $4(3.9)$ & $6(5.1)$ \\
\hline
\end{tabular}

${ }^{*}$ COVID-19 severity: maximum oxygen required during initial hospitalisation. Mild: nasal cannula, venturi mask or no supplemental oxygen; moderate: non-rebreather mask, high-flow nasal cannula or non-invasive ventilation; severe: intubation. †Reduced mobility: need for an ambulatory assist device, ongoing physical therapy or reported difficulty with ambulation. $\ddagger$ Reduced independence: inability to live alone without support from a home health aide, nurse or family member. 
Table 5 Disposition of patients within the community prior to admission, on discharge and at each 3-month time period

\begin{tabular}{lcccc}
\hline Characteristics & $\begin{array}{l}\text { Admitted from } \\
\mathbf{N = 1 1 9 0 ( \% )}\end{array}$ & $\begin{array}{l}\text { Discharged to } \\
\mathbf{N = 1 1 9 0}(\%)\end{array}$ & $\begin{array}{l}\text { 3 months } \\
\mathbf{n = 5 7 4}(\%)\end{array}$ & $\begin{array}{l}\mathbf{6} \text { months } \\
\mathbf{n = 4 4 5}(\%)\end{array}$ \\
\hline Home & $1066(89.6)$ & $727(61.1)$ & $454(79.1)$ & $386(86.8)$ \\
Nursing facility or rehabilitation & $86(7.2)$ & $157(13.2)$ & $21(3.7)$ & $32(7.2)$ \\
Hospital & $17(1.4)$ & $20(1.7)$ & $9(1.6)$ & $11(2.5)$ \\
Shelter or undomiciled & $21(1.8)$ & $16(1.3)$ & $31(5.4)$ & $6(2.2)$ \\
Death or hospice & & $270(22.7)$ & & $6(1.3)$ \\
\hline
\end{tabular}

its best uses. ${ }^{36}$ In particular, as the need for post-COVID-19 clinics increases, expanding telehealth availability would allow more patients to be evaluated, especially those who are unable or unwilling to attend an inperson visit. ${ }^{10}$

This study had a number of limitations. First, as a retrospective cohort study, data collection was based on clinician documentation and relied on patient recall and reporting of symptoms rather than a validated standardised questionnaire, actively given at all patient encounters or at some scheduled period. Symptoms were collected from self-reports without any objective measurements and symptoms were not evaluated for their chronicity prior to hospitalisation. Although this observed pattern did not remain at 6 months, outcomes may have been caused by underlying comorbidities rather than COVID-19. Importantly, given the small number of mental health visits, data on the psychiatric and emotional toll of COVID-19 are likely to be under-reported in this cohort. Second, the accuracy of the data collection relied on data abstraction through manual chart review. This limitation was mitigated through the addition of a dedicated quality control abstractor and a review of a random subsample of the abstracted data by the lead clinician. Third, this study is limited to patients whose care was documented at one academic, quaternary care hospital in New York City. As the data were collected via chart abstraction, only follow-up encounters within the NYP/CUIMC system were included and only 570 of the surviving 929 individuals from the initial cohort $(61 \%)$ had a follow-up encounter within our institution. Data from follow-up visits within community clinics, other private hospitals and the public hospital system were not able to be collected. Notably, the initiation of a systemic programme providing patients with discharge appointments did not alter the rates of follow-up significantly. Because patients were not actively followed, follow-up results are at higher risk of selection bias from symptomatic patients engaging more than patients who fully recovered. Similarly, the rates of symptoms among patients without comorbidities may have been overestimated given that patients without follow-up encounters had fewer medical comorbidities. The decision to exclude patients without encounters from our analysis may have also overestimated the frequency of symptoms and morbidity outcomes. However, our methodology of reporting only persistent symptoms or morbidity outcomes within each time period instead of every occurrence likely led to underestimations of both. Furthermore, by only including those persistent variables, we were therefore unable to calculate the duration of symptoms or morbidity after COVID-19 hospitalisation. Lastly, while our study found higher prevalence of specialty visits at both the 3-month and 6-month follow-up periods, this finding may be due to the inability to capture community primary care visits and the high density of specialists within our institution.

Despite these limitations, our study has demonstrated the long-term morbidity associated with higher severity of initial COVID-19 presentation; a significant number of patients require nursing care after admission, and cardiopulmonary, generalised and neuropsychiatric symptoms can persist up to 6 months following initial illness. These findings highlight the burden of COVID-19 on both patients and healthcare resources in our community. Given the persistence of symptoms and degree of morbidity up to 6 months after diagnosis, continued follow-up of patients with COVID-19 is essential to understand the long-term sequelae of this novel disease.

\section{Author affiliations}

${ }^{1}$ Medicine, Infectious Diseases, Columbia University Irving Medical Center, New York City, New York, USA

${ }^{2}$ Medicine, Columbia University Irving Medical Center, New York City, New York, USA ${ }^{3}$ Columbia University Vagelos College of Physicians and Surgeons, New York City, New York, USA

${ }^{4}$ Biomedical Informatics, Columbia University Irving Medical Center, New York City, New York, USA

${ }^{5}$ Translational Data Science and Informatics, Geisinger, Danville, Pennsylvania, USA

Acknowledgements We would like to acknowledge the data in the COVID-CARE database based at NYP/CUIMC, Division of Infectious Diseases.

Contributors SMS is the first author. JEZ and DC share senior authorship. Study conception and design: SMS, JEZ, DC, LP, MGA, SLB, CLS, JRT, RC. Manuscript composition: SMS, MAA, CD, DC. Acquisition, analysis or interpretation of data: SMS, JEZ, DC, LP, MAA, ALI, SYR-J, AMK, DL, AAH, LMZ, KEL, KG, MCL, NC, CPS, SSM, ELM, HS. Review and revision of the manuscript for important intellectual content: SMS, LP, MAA, DC, DAT, ALI, SYR-J, AMK, DL, AAH, LMZ, KEL, KG, MCL, NC, CPS, SSM, ELM, HS, GH, KN, RC, MTY, MES, JEZ, DC. Study supervision and guarantors of the study: SMS, JEZ, DC. The corresponding author attests that all listed authors meet the authorship criteria and that no others meeting the criteria have been omitted.

Funding Research reported in this publication was supported by the National Institute of Allergy and Infectious Diseases of the National Institutes of Health under award number K23Al150378 (JEZ), L30Al133789 (JEZ), UM1Al069470 and supplement to the award (DAT, MES, JEZ). The content is solely the responsibility of the authors and does not necessarily represent the official views of the National Institutes of Health. SMS is supported by T32Al100852-08. KEL is supported by T32DK083256-12S1. GH is supported by R01LM006910. No funding organisation or sponsor was involved in the study design, data collection or analysis, manuscript composition, or the decision to submit for publication.

Competing interests GH reports grants from Janssen Research, outside the submitted work.

Patient consent for publication Not required. 
Ethics approval The Columbia University Irving Medical Center Institutional Review Board approved this study under protocol AAAS9622.

Provenance and peer review Not commissioned; externally peer reviewed.

Data availability statement Data are available upon reasonable request. Requests for the statistical code and data set can be made to the corresponding author.

Supplemental material This content has been supplied by the author(s). It has not been vetted by BMJ Publishing Group Limited (BMJ) and may not have been peer-reviewed. Any opinions or recommendations discussed are solely those of the author(s) and are not endorsed by BMJ. BMJ disclaims all liability and responsibility arising from any reliance placed on the content. Where the content includes any translated material, BMJ does not warrant the accuracy and reliability of the translations (including but not limited to local regulations, clinical guidelines, terminology, drug names and drug dosages), and is not responsible for any error and/or omissions arising from translation and adaptation or otherwise.

Open access This is an open access article distributed in accordance with the Creative Commons Attribution Non Commercial (CC BY-NC 4.0) license, which permits others to distribute, remix, adapt, build upon this work non-commercially, and license their derivative works on different terms, provided the original work is properly cited, appropriate credit is given, any changes made indicated, and the use is non-commercial. See: http://creativecommons.org/licenses/by-nc/4.0/.

\section{ORCID iDs}

Sherif M Shoucri http://orcid.org/0000-0002-6290-2320

Matthew A Adan http://orcid.org/0000-0003-2515-8982

Alexandria Lauren Irace http://orcid.org/0000-0003-4758-3146

Kate E Lee http://orcid.org/0000-0002-0171-7121

Michael G Argenziano http://orcid.org/0000-0003-3374-9037

\section{REFERENCES}

1 Johns Hopkins Coronavirus Resource Center, 2020. Available: https://coronavirus.jhu.edu

2 Cdc COVID data Tracker, 2020. Available: https://covid.cdc.gov/ covid-data-tracker/\#cases_casesper100klast7days

3 Argenziano MG, Bruce SL, Slater CL, et al. Characterization and clinical course of 1000 patients with coronavirus disease 2019 in New York: retrospective case series. BMJ 2020;369:m1996.

4 Leung TYM, Chan AYL, Chan EW, et al. Short- and potential longterm adverse health outcomes of COVID-19: a rapid review. Emerg Microbes Infect 2020;9:2190-9.

5 Long COVID: let patients help define long-lasting COVID symptoms. Nature 2020;586:170.

6 Mahase E. Covid-19: What do we know about "long covid"? BMJ 2020;370:m2815.

7 Logue JK, Franko NM, McCulloch DJ, et al. Sequelae in adults at 6 months after COVID-19 infection. JAMA Netw Open 2021;4:e210830.

8 Writing Committee for the COMEBAC Study Group, Morin L, Savale $\mathrm{L}$, et al. Four-Month clinical status of a cohort of patients after hospitalization for COVID-19. JAMA 2021;325:1525-34.

9 Havervall S, Rosell A, Phillipson M, et al. Symptoms and functional impairment assessed 8 months after mild COVID-19 among health care workers. JAMA 2021;325:2015.

10 Nalbandian A, Sehgal K, Gupta A, et al. Post-Acute COVID-19 syndrome. Nat Med 2021;27:601-15.

11 Huang C, Huang L, Wang Y, et al. 6-Month consequences of COVID-19 in patients discharged from Hospital: a cohort study. Lancet 2021;397:220-32.

12 Vasarmidi E, Tsitoura E, Spandidos DA, et al. Pulmonary fibrosis in the aftermath of the COVID-19 era (review). Exp Ther Med 2020;20:2557-60.

13 Calabrese F, Pezzuto F, Fortarezza F, et al. Pulmonary pathology and COVID-19: lessons from autopsy. The experience of European pulmonary pathologists. Virchows Arch 2020;477:359-72.
14 Wang F, Kream RM, Stefano GB. Long-Term respiratory and neurological sequelae of COVID-19. Med Sci Monit 2020;26:e928996.

15 Salehi S, Reddy S, Gholamrezanezhad A. Long-Term pulmonary consequences of coronavirus disease 2019 (COVID-19). J Thorac Imaging 2020;35:W87-9.

16 Shaw B, Daskareh M, Gholamrezanezhad A. The lingering manifestations of COVID-19 during and after convalescence: update on long-term pulmonary consequences of coronavirus disease 2019 (COVID-19). Radiol Med 2021;126:40-6.

17 Becker RC. Anticipating the long-term cardiovascular effects of COVID-19. J Thromb Thrombolysis 2020;50:512-24.

18 Puntmann VO, Carerj ML, Wieters I, et al. Outcomes of cardiovascular magnetic resonance imaging in patients recently recovered from coronavirus disease 2019 (COVID-19). JAMA Cardiol 2020;5:1265-73.

19 Yancy CW, Fonarow GC. Coronavirus disease 2019 (COVID-19) and the Heart-Is heart failure the next chapter? JAMA Cardiol 2020;5:1216-7.

20 Bridwell R, Long B, Gottlieb M. Neurologic complications of COVID-19. Am J Emerg Med 2020;38:1549.e3-1549.e7.

21 Troyer EA, Kohn JN, Hong S. Are we facing a crashing wave of neuropsychiatric sequelae of COVID-19? neuropsychiatric symptoms and potential immunologic mechanisms. Brain Behav Immun 2020;87:34-9.

22 Kemp HI, Corner E, Colvin LA. Chronic pain after COVID-19: implications for rehabilitation. Br J Anaesth 2020;125:436-40.

23 Rhea EM, Logsdon AF, Hansen KM, et al. The S1 protein of SARS-CoV-2 crosses the blood-brain barrier in mice. Nat Neurosci 2021;24:368-78.

24 Taquet M, Luciano S, Geddes JR, et al. Bidirectional associations between COVID-19 and psychiatric disorder: retrospective cohort studies of 62354 COVID-19 cases in the USA. The Lancet Psychiatry 2021;8:130-40.

25 Ding X, Xu J, Zhou J, et al. Chest CT findings of COVID-19 pneumonia by duration of symptoms. Eur J Radiol 2020;127:109009.

26 Carfi A, Bernabei R, Landi F, et al. Persistent symptoms in patients after acute COVID-19. JAMA 2020;324:603-5.

27 Chopra V, Flanders SA, O'Malley M, et al. Sixty-Day outcomes among patients hospitalized with COVID-19. Ann Intern Med 2021;174:576-8.

28 Harris PA, Taylor R, Minor BL, et al. The REDCap Consortium: building an international community of software platform partners. $J$ Biomed Inform 2019;95:103208.

29 Harris PA, Taylor R, Thielke R, et al. Research electronic data capture (REDCap)-A metadata-driven methodology and workflow process for providing translational research informatics support. J Biomed Inform 2009;42:377-81.

30 Ye S, Hiura G, Fleck E, et al. Hospital readmissions after implementation of a discharge care program for patients with COVID-19 illness. J Gen Intern Med 2021;36:722-9.

31 R Core Team. R: a language and environment for statistical computing. Vienna, Austria: R Foundation for Statistical Computing, 2020. https://www.R-project.org/

32 Zhao Y-miao, Shang Y-min, Song W-bin, Zhao Y, Shang Y, Song $\mathrm{W}$, et al. Follow-Up study of the pulmonary function and related physiological characteristics of COVID-19 survivors three months after recovery. EClinicalMedicine 2020;25:100463.

33 Herridge MS, Cheung AM, Tansey CM, et al. One-Year outcomes in survivors of the acute respiratory distress syndrome. $N$ Engl J Med 2003;348:683-93.

34 Luyt C-E, Combes A, Becquemin M-H, et al. Long-term outcomes of pandemic 2009 influenza $A(H 1 N 1)$-associated severe ARDS. Chest 2012;142:583-92.

35 Demeke HB, Pao LZ, Clark H, et al. Telehealth Practice Among Health Centers During the COVID-19 Pandemic - United States, July 11-17, 2020. MMWR Morb Mortal Wkly Rep 2020;69:1902-5.

36 Shah ED, Amann ST, Karlitz JJ. The time is now: a guide to sustainable telemedicine during COVID-19 and beyond. Am J Gastroenterol 2020;115:1371-5. 